\title{
Dosimetric Accuracy of an HDR Brachytherapy Treatment Planning System for Different Irradiation Lengths with Monte Carlo Simulation
}

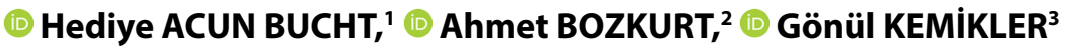 \\ 'Department of Biophysics, Harran University Faculty of Medicine, Şanlıurfa-Turkey \\ ${ }^{2}$ Department of Biomedical Engineering, Akdeniz University Faculty of Engineering, Antalya-Turkey \\ ${ }^{3}$ Department of Medical Physics, Istanbul University Institute of Oncology, İstanbul-Turkey
}

\begin{abstract}
OBJECTIVE
The purpose of the present study was to verify calculated dose rate profiles of Ir-192 for four different irradiation lengths and various numbers of dwell positions by using treatment planning system (TPS) calculations and Monte Carlo (MC) simulations.

\section{METHODS}

Dose rate profiles per air-kerma strength in $\mu \mathrm{Gy} \mathrm{m} \mathrm{m}^{2} / \mathrm{h}$ (abbreviated as $\mathrm{U}$ ) were calculated on transverse axis for irradiation lengths of $2,3,4$, and $5 \mathrm{~cm}$ by Nucletron Oncentra TPS. The same irradiation scenarios were simulated with Monte Carlo N-Particle (MCNP) Transport Code. The MC and TPS calculated dose rate profiles were compared for all settings.
\end{abstract}

\section{RESULTS}

The difference between the calculated dose rate profiles by MC and TPS was found within $2 \%$ at an interval of $2 \mathrm{~mm}-8 \mathrm{~cm}$ away from the source for all irradiation lengths. The largest discrepancy of $5.2 \%$ was computed at a distance of $10 \mathrm{~cm}$ from the source for $5 \mathrm{~cm}$ irradiation length.

\section{CONCLUSION}

There is a good consistency between the calculated dose rate profiles by MCNP and Oncentra TPS. The deviation between the calculated dose rate values slightly increases as the distance from the source center increases $>5 \mathrm{~cm}$ from the source, and its quantity depends on the number of dwell positions.

Keywords: Dose rate profile; HDR brachytherapy; Monte Carlo; TPS.

Copyright $\odot$ 2019, Turkish Society for Radiation Oncology

\section{Introduction}

Brachytherapy is classified into three types in accordance with dose rate from the radioactive source in use. The dose rate of the source is expected to be $>12$ Gy/h in HDR brachytherapy where Ir-192 isotope is encapsulated into a cylindrical shape in after-loading systems.[1,2,3] The clinical application of HDR brachytherapy involves obtaining dose distribution from the calculation algorithm of a commercial treatment planning system (TPS). Nowadays, the majority of HDR brachytherapy TPSs are based on the American Association of Physicists in Medicine (AAPM) Task Group report 43 (TG-43) dose calculation formalism, whereas previously, a classical calculation was used.[4] Classical dose calculation is based on 
point source approximation and calculates the dose rate by the use of the source activity, the tissue attenuation coefficient, and the exposure rate. In this method, the dose around the elongated source is computed by Sievert integration procedure as the summation of the contributions from point source elements. This formalism does not take into account the properties of the source, such as encapsulation and internal construction. On the other hand, TG-43 dosimetry formalism uses some dosimetry parameters that depend on source properties to calculate the dose rate.[4,5] The AAPM proposes that these dosimetry parameters for brachytherapy sources, such as air-kerma strength $\left(S_{K}\right)$, dose rate constant $(\Lambda)$, geometry factor $G(r, \theta)$, radial dose function $g(r)$, and two-dimensional (2D) anisotropy factor $F(r, \theta)$, could be measured experimentally or calculated through Monte Carlo simulation (MCS).[5] MCS is a reliable and well-known calculation method for calculation of the brachytherapy dosimetry parameters since the source could be modeled realistically. There are studies about the derivation of the TG-43 dosimetry parameters with different MC codes in the literature.[2,6,7] The dose accuracy of a radioactive source could be achieved experimentally by the use of appropriate dosimeters. However, it is difficult to position the detector with an acceptable uncertainty due to the high-dose gradient at close distances around the source.[3,8] In addition, some properties of the dosimeters, such as linearity, material composition, energy dependence, and spatial resolution, should be taken into account with regard to dose accuracy as well. Thermoluminescent dosimeters and Gafchromic films are the most common dosimeters in brachytherapy owing to their distinct properties.[8]

HDR has become the most commonly used treatment modality in clinical brachytherapy due to its advantages, such as rapid dose reduction, less doses to the surrounding anatomical structures, and quick implementation and personal radiation safety. Dose distribution in HDR brachytherapy is calculated by commercial proprietary TPS software packages that include complicated algorithms to perform the evaluation because of the presence of hot spots and highdose gradients at close distances to the source. The source moves in steps through the applicator to attain an intended irradiation length in HDR brachytherapy. The number of dwell positions, the source step size, and the dwell times can be adjusted according to the target volume as part of the TPS optimization procedure. $[9,10]$ The prescribed dose is delivered in sever- al fractions to the patient, and accordingly, the dose amount of each treatment is relatively high. Therefore, the dosimetric accuracy of TPS in HDR brachytherapy is quite essential. There are many studies that were conducted with MCS in the literature related to the verification of dose distribution generated by different TPSs around various sources and applicators.[11-13] Naseri et al. performed dosimetric verification of an HDR TPS, based on the Sievert integration method for dose calculation, using MCNP4C code for three different Co-60 sources.[14] Toossi et al. performed the dosimetric validation of an HDR TPS, which uses Sievert integration for dose calculation, for irradiation lengths of 8 and $10 \mathrm{~cm}$ by the movement of the Co-60 source along the treatment axis.[15] Hadad et al. performed dosimetric verification of a TG-43 based on TPS for a nasopharynx case by means of MCS.[16] To the best of our knowledge, there is no study regarding dosimetric validation of an HDR TPS, which is based on TG-43 formalism, for various irradiation lengths using MCS in the literature. The aim of the present study was to calculate the transverse dose rate profiles of various irradiation lengths in the range of $2 \mathrm{~cm}-5 \mathrm{~cm}$, which are the most commonly used source lengths clinically, with MCS and to compare the simulation results with those calculated by Nucletron Oncentra TPS. Accordingly, it can be possible to investigate the effect of the irradiation length on the dose difference between MCS and TPS.

\section{Materials and Methods}

\section{Nucletron Oncentra Treatment Planning System}

The calculation algorithm of Nucletron (Nucletron; Elekta Company, Elekta AB, Stockholm, Sweden) Oncentra MasterPlan TPS (version 3.2) is based on the AAPM TG-43 formalism that performs the dose rate calculations in a water medium with fixed size for radiation scattering and makes an assumption of the encapsulated brachytherapy source as cylindrically symmetric. This particular TPS has the concept of virtual dose rate to generate the isodose contours around the irradiation volume since the intended dose distribution is created by the stepwise movement of the source. When the source moves to the next step, the dose rate of any arbitrary point around a dwell position decreases. Therefore, the summation of the dose from each dwell position to any point of interests generates the virtual dose rate at that specific point. Each dwell position has a weight ranging between 0.0 and 1.0 in Oncentra TPS that can be changed according 
to the clinical goal. In the present study, four different irradiation lengths of 2, 3, 4, and $5 \mathrm{~cm}$ were created in Oncentra TPS. The step size of the source was set to $0.25 \mathrm{~mm}$; therefore, the numbers of dwell positions were $9,13,17$, and 21 for irradiations of 2,3 , 4, and 5 $\mathrm{cm}$, respectively.

The activity of the Ir-192 source was 7.965 Curie, and the irradiation times were generated through the calculation algorithm of TPS based on source activity and dose normalization. All dwell positions were weighted equally, and a dose of $10 \mathrm{~Gy}$ was normalized to $1 \mathrm{~cm}$ away from the source for each irradiation scenario. Therefore, the total dwell time of each case was different as various numbers of dwell positions were contained within.

Absolute dose rate profiles were calculated in the transverse directions from the center of the source at a distance of $\pm 10 \mathrm{~cm}$ for every irradiation length. The calculated dose values by TPS were normalized to the air-kerma strength of the source in use stated in the certificate of the vendors for comparing them with the simulation results.

\section{MCNP Transport Code}

Monte Carlo N-Particle (MCNP) Transport Code is a MCS code used for computation of radiation interactions including photons, neutrons, and electrons in any desired combinations. In this method, individual radiation particle's characteristics, such as energy, position, flight direction, track length, and the energy fluence on a plane or the dose deposition in a voxel, could be calculated by the use of the random number engine. [17] This simulation code was used in the study for modeling the Ir-192 source and calculation of the dose around the mentioned irradiation lengths.

In the present study, Nucletron HDR ${ }^{192} \mathrm{Ir} \mathrm{Mi}$ croselectron v2 (model mHDR-v2) is the source remote controlled by the after-loading device. All components of the source with geometrical properties and material compounds were realistically modeled with MCNP. Nucletron mHDR-v2 source consists of a central cylindrical Iridium core (density: $22.42 \mathrm{~g} / \mathrm{cc}$, diameter: $0.65 \mathrm{~mm}$, and height: $3.6 \mathrm{~mm}$ ) and a surrounding outer capsule (AISI-316; outer diameter: $0.9 \mathrm{~mm}$ and thickness: $0.125 \mathrm{~mm}$ ) made of stainless steel (density: $7.92 \mathrm{~g} / \mathrm{cc}$ and weight fraction: $1 \% \mathrm{Si}, 17 \% \mathrm{Cr}, 2 \% \mathrm{Mn}$, $65.5 \% \mathrm{Fe}, 12 \% \mathrm{Ni}$, and $2.5 \% \mathrm{Mo}$ ).

The source strength of a radioactive source is specified by the air-kerma strength $\left(S_{K}\right)$ in brachytherapy. Air-kerma strength is one of the TG-43 dosimetry parameters, and its quantity varies depending on the con- tent and geometry of the source. Therefore, SK should be calculated for every source in question. In the literature, the dose rate around the source is usually specified by normalizing to the air-kerma strength (SK) in $\mathrm{U}\left(1 \mathrm{U}=\mu \mathrm{Gy} \mathrm{m} \mathrm{m}^{2} / \mathrm{h}\right) .[6,18,19]$ In the present study, 36 spherical detectors were located at the points aligned with equal angle intervals of $10^{\circ}$ on a circle with a 1 $\mathrm{m}$ radius around the source (in upright position) to compute the air-kerma strength of the involved source. Air-kerma calculations were performed in vacuum medium as suggested by the TG-43 report.

The source lies and moves along the y-axis in the simulations with MCNP as in Oncentra TPS. The irradiation scenarios of $2,3,4$, and $5 \mathrm{~cm}$ were created in a water phantom of $40 \times 40 \times 40 \mathrm{cc}$ (density: $1 \mathrm{~g} / \mathrm{cc}$ and weight fraction: $11.19 \% \mathrm{H}$ and $88.81 \% \mathrm{O}$ ) and were surrounded by spherical dose cells made of water with a radius of $0.25 \mathrm{~cm}$. Dose cells were positioned along the central plane of the associated irradiation lengths to scan an area of $10 \times 10 \mathrm{~cm} 2$. An air sphere (density: $0.001205 \mathrm{~g} / \mathrm{cc}$, weight fraction: $0.0124 \% \mathrm{C}, 75.5268 \%$ $\mathrm{N}, 23.1781 \% \mathrm{O}$, and $1.2827 \% \mathrm{Ar}$, and radius: $50 \mathrm{~cm}$ ) was defined around the water phantom. Figure $1 \mathrm{de}-$ picts a plot of the geometry in question as drawn by the MCNP code. All parameters of the mentioned irradiation scenarios, such as the coordinates and the number of dwell positions, the step size of the source, and the dwell times, in MCNP were set to the same quantities with those in TPS. The dose in each cell was obtained as the summation of the doses from each dwell position.

Only photons were taken into account with regard to radiation transport in the simulations without specifying any flight directions of the source particles ( $4 \pi$ isotropic distribution). The energy spectrum of the Ir-192 source was defined as containing both the gamma and the X-ray lines of the isotope. The energy deposition ( $\mathrm{MeV} / \mathrm{g} /$ particle) tally (F6) was used in the simulations to record the absolute dose in the point of interests. The statistical uncertainty was achieved to be $<2 \%$ for all sets of simulations as a result of running 100 million photon histories per set.

\section{Results}

The mean value of air-kerma strength (SK) was computed as $9.79 \times 10^{-8}\left(9.465 \times 10^{-8}-9.832 \times 10^{-8}\right)$ U/Bq using MCNP simulations. The air-kerma strength per activity at a point $1 \mathrm{~m}$ away along the transverse axis of the source was calculated as $9.86 \times 10^{-8} \mu \mathrm{Gy} \mathrm{m} / \mathrm{h} / \mathrm{Bq}$. All absolute doses obtained by MCNP were normal- 


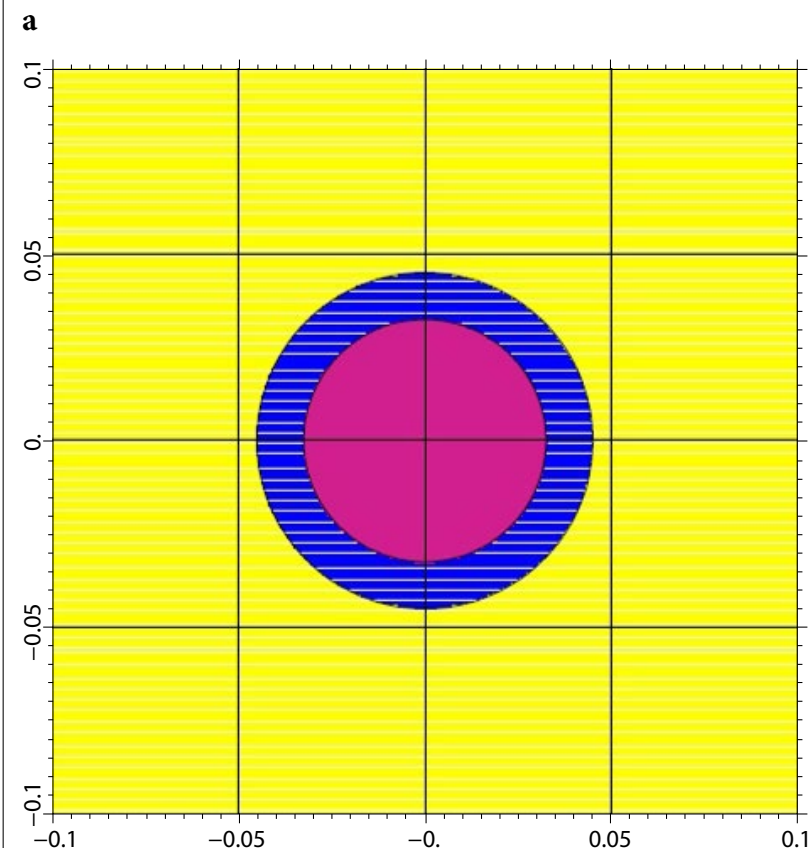

c

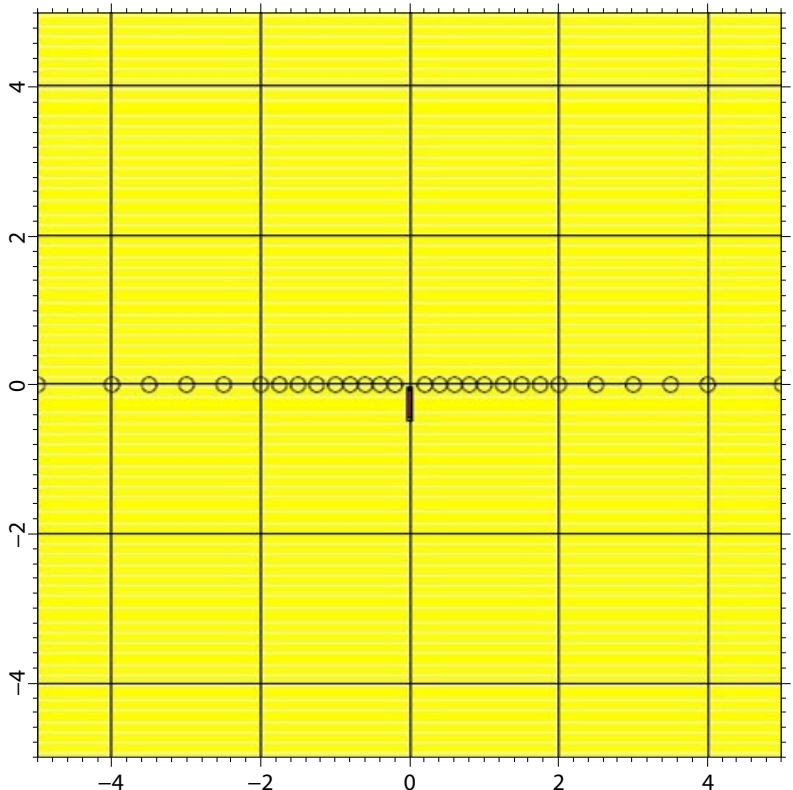

b

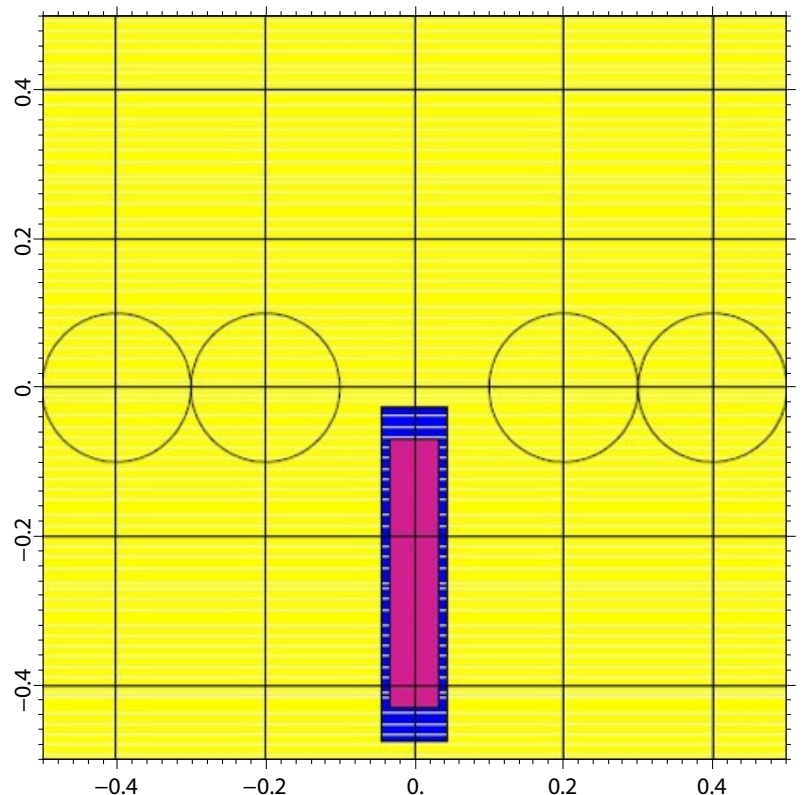

d

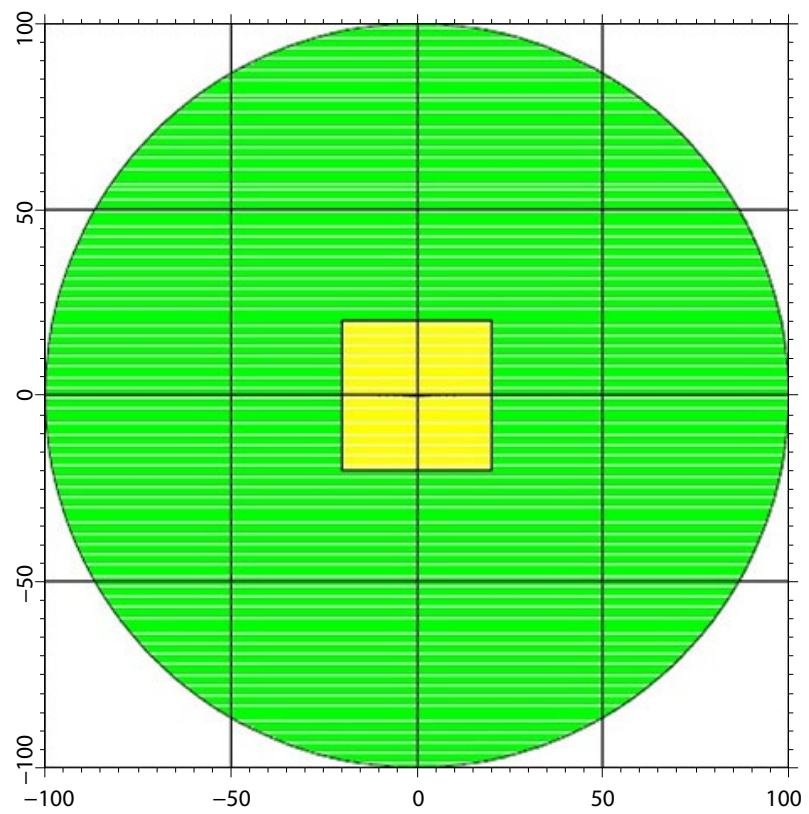

Fig. 1. MCNP plot of the source, phantom, and detectors in question. All dimensions are in $\mathrm{cm}$. $(\mathrm{a}) \mathrm{xz}(\mathrm{y}=0) \mathrm{phantom}+$ transverse view of the source. (b) $x y(z=0)$ phantom + coronal view of the source. $(c) x y(z=0)$ phantom + source + detectors. $(\mathrm{d}) \mathrm{xy}(\mathrm{z}=0)$ complete geometry.

ized by the last mentioned SK value since it was the SK value of the TG-43 reference point.

The percentage difference between the calculated doses by MCNP and TPS at the points of interest was obtained according to the following equation:
Difference $(\%)=\frac{M C N P-T P S}{M C N P} \times 100$

The dose rate profiles per air-kerma strength, calculated by MCNP and TPS, on the central axis of the ir- 

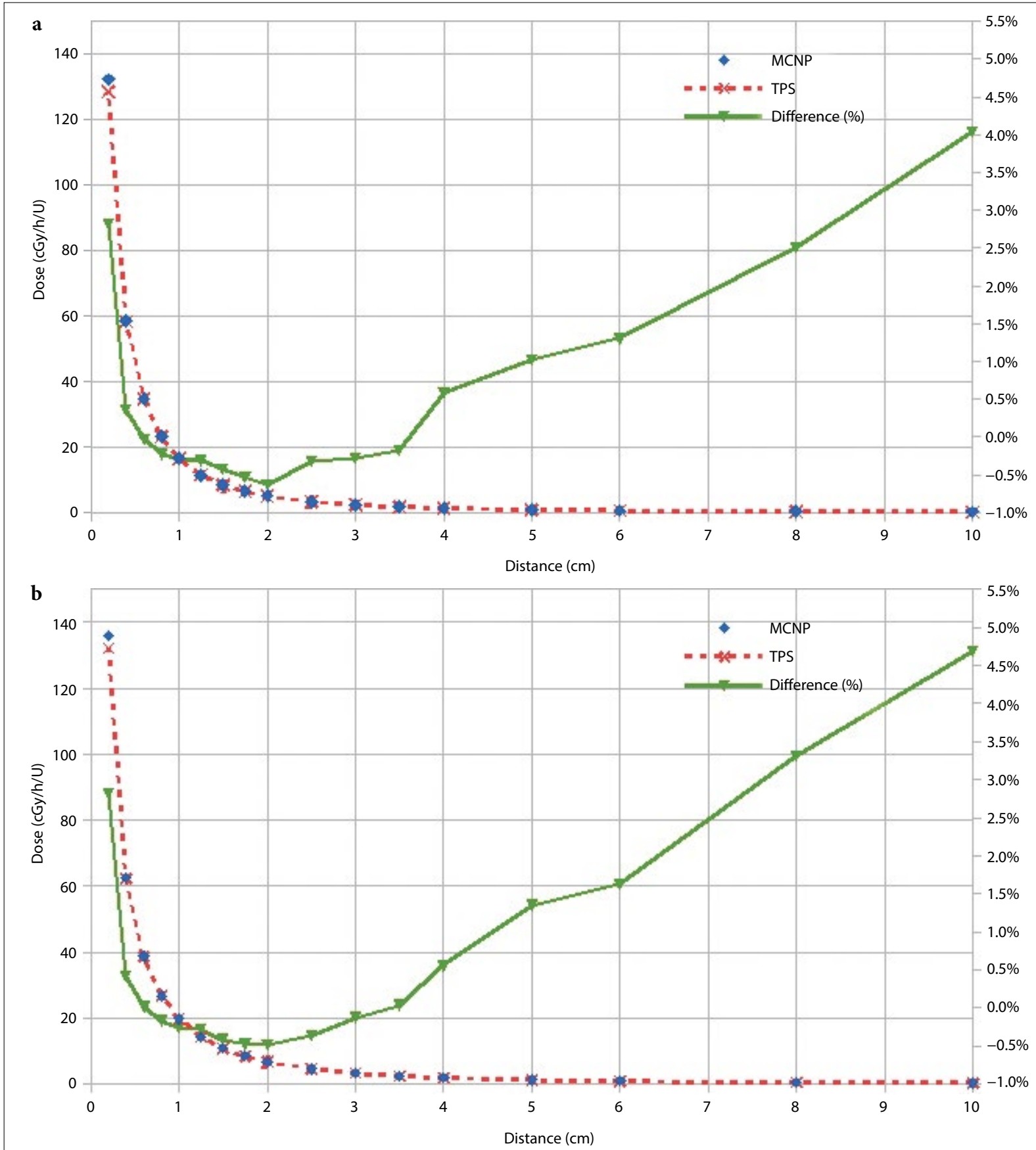

Fig. 2. Comparison of absolute dose rate profiles, per U, calculated by MCNP and Oncentra TPS on the central axis of the irradiation length along the $\mathrm{x}$-axis (perpendicular to the source movement direction). Irradiation lengths of (a) 2 $\mathrm{cm}$, (b) $3 \mathrm{~cm}$, (c) $4 \mathrm{~cm}$, and (d) $5 \mathrm{~cm}$.

radiation length along the $\mathrm{x}$-axis (perpendicular to the source movement direction) are shown in Figures 2a, $\mathrm{b}, \mathrm{c}$, and $\mathrm{d}$ for irradiation scenarios of $2,3,4$, and $5 \mathrm{~cm}$ lengths, respectively.

Dose rates per U calculated by TPS were found to be $2.82 \%, 2.82 \%, 2.80 \%$, and $2.73 \%$ lower than those computed by MCNP at close distances $(\mathrm{x}=2 \mathrm{~mm})$ to the source center for $2,3,4$, and $5 \mathrm{~cm}$ irradiation scenarios, respectively. The difference between MCNP and TPS calculated dose rates is $<1 \%$ at distances farther than $6 \mathrm{~mm}$ away from the source center $(\mathrm{x} \geq 6$ $\mathrm{mm}$ ) and $>1 \%$ beyond $5 \mathrm{~cm}$ from the irradiation cen- 

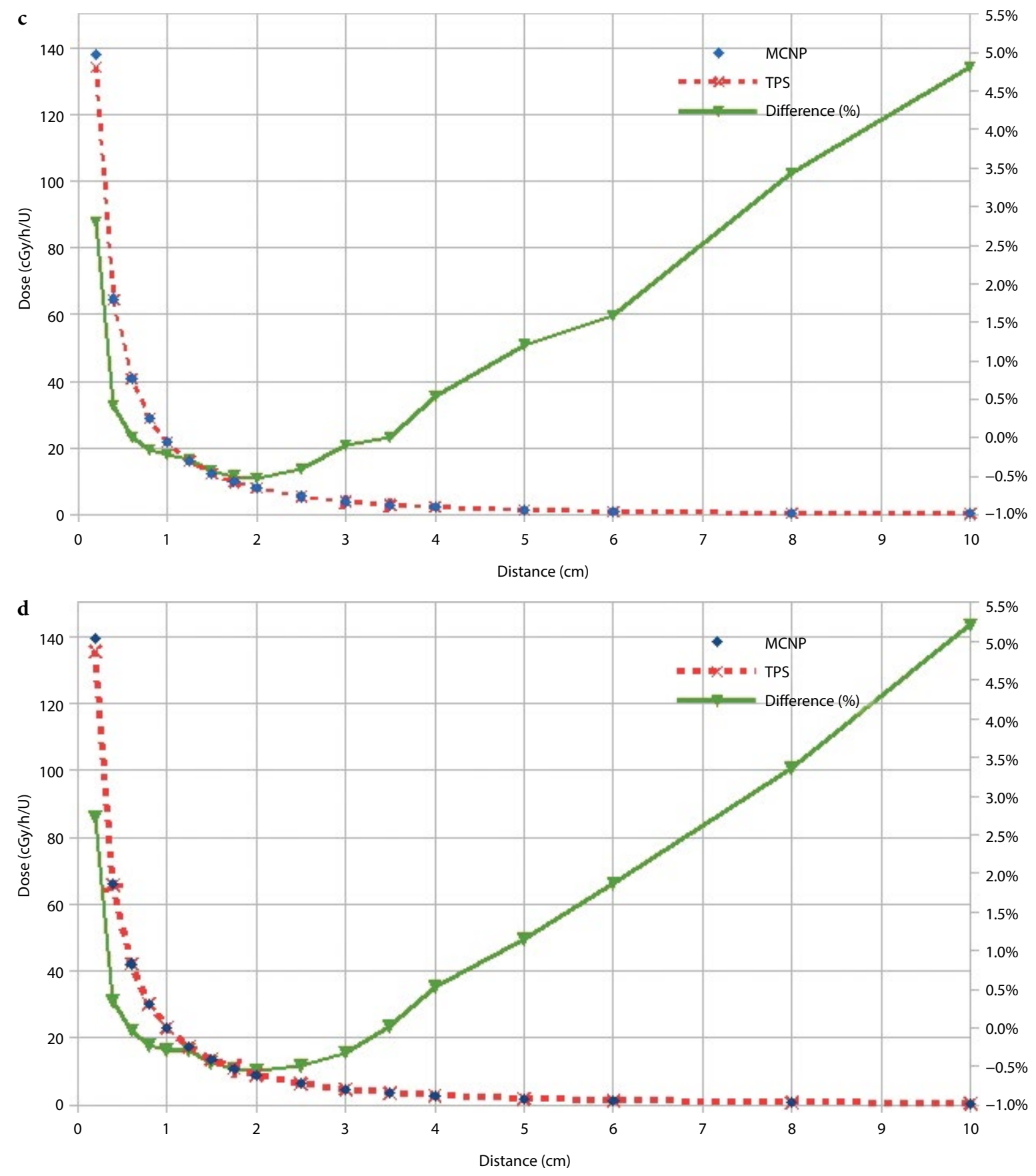

Fig. 2. Comparison of absolute dose rate profiles, per U, calculated by MCNP and Oncentra TPS on the central axis of the irradiation length along the $\mathrm{x}$-axis (perpendicular to the source movement direction). Irradiation lengths of (a) 2 $\mathrm{cm}$, (b) $3 \mathrm{~cm}$, (c) $4 \mathrm{~cm}$, and (d) $5 \mathrm{~cm}$.

ter. The differences between TPS and MCNP were calculated as $2.51 \%, 3.31 \%, 3.44 \%$, and $3.35 \%$ at $8 \mathrm{~cm}$ and $4.03 \%, 4.68 \%, 4.82 \%$, and $5.21 \%$ at $10 \mathrm{~cm}$ away from the source center for irradiation lengths of 2 , 3,4 , and $5 \mathrm{~cm}$, respectively. The dose differences be- tween MCNP and TPS have been observed to increase with irradiation length at distances farther than $6 \mathrm{~cm}$ from the center. However, the dose differences for all irradiation scenarios are almost equal to each other at the points near the source. 


\section{Discussion}

It is quite important to accomplish the accuracy of dose distribution as calculated by TPS in high-dose rate brachytherapy due to the presence of a steep dose gradient around the irradiated site and the considerable high dose per fraction. MC can be used for calculation of TG-43 dosimetry parameters and doses around the radioactive source by taking into account the radiation interactions for involved source and medium. In the present study, dosimetric validation of Nucletron Oncentra TPS was performed with MCNP simulation code for irradiation scenarios of $2,3,4$, and $5 \mathrm{~cm}$ source lengths.

There have been published studies regarding MC calculation of TG-43 dosimetry parameters of Nucletron mHDR-v2 Ir-192 source. Granero et al. modeled Nucletron mHDR-v2 and mHDR-v2r (new design) Ir-192 sources with three different MC codes, namely MCNP5, PENELOPE2008, and GEANT4, to calculate and compare the TG-43 dosimetry parameters that are dose rate constant, radial dose function, and $2 \mathrm{D}$ anisotropy factor.[20] They did not observe any noticeable difference between the dosimetry parameters of the above-mentioned Nucletron Ir-192 source beyond $0.25 \mathrm{~cm}$ distance $(\mathrm{r} \geq 0.25 \mathrm{~cm})$ from the source center. In another published study, air-kerma rate and other TG-43 dosimetry parameters were calculated for the mentioned sources in the previous study with PENELOPE2008.[21] Lopez et al. calculated the air-kerma rate of Nucletron Microselectron mHDR-v2 and mHDR-v2r to be $9.78 \times 10^{-8} \mathrm{U} / \mathrm{Bq}$ and $9.86 \times 10^{-8} \mathrm{U} /$ $\mathrm{Bq}$, respectively, at the point $1 \mathrm{~m}$ away from the source on the perpendicular direction to the axis where the source lies $(\theta=\pi / 2)$. In our study, the air-kerma rate of Nucletron mHDR-v2 Ir-192 source was computed as $9.86 \times 10^{-8} \mathrm{U} / \mathrm{Bq}$ at the same point of their study. There is a good consistency between our study and their study as our calculated air-kerma rate differs $0.8 \%$ from the value calculated by Lopez et al.[21]

MC technique has been used frequently for validation of various TPSs in addition to the calculation of TG-43 dosimetry parameters of different HDR brachytherapy sources. Naseri et al. calculated dose distribution around three different Co-60 sources with MCNP4C code. Thereafter, the simulated results were compared with those calculated by a classical calculation based on an HDR TPS. They found a large deviation between MC and TPS calculated isodose lines near the source region.[14] They attributed this difference to the high-dose gradient at close region around the HDR source. Hadad et al. studied the HDR treatment plan of a patient with nasopharynx cancer by using computed tomography (CT) images of a patient and a water equivalent phantom with DOSXYZnrc software. The dose distribution around the source in the treatment plan was calculated in water equivalent and virtual phantom obtained out of the patient CT images separately. The simulated values in water equivalent phantom were compared with the dose distribution calculated by Oncentra TPS, which is based on the TG-43 formalism for the same irradiation parameters. In their study, two isodose values of $0.25 \mathrm{~Gy}$ and $1 \mathrm{~Gy}$ were calculated with MC and TPS. They concluded that Oncentra TPS calculates lower dose values at the near source region and higher ones at further distances from the source than MC.[16] The dose rate distribution of the new design Microselectron Nucletron HDR Ir-192 source in the near source region has also been studied, and dose rate has been observed to be affected by the non-equilibrium of the charged particle at close distances $(0.5 \leq \mathrm{r} \leq 2 \mathrm{~mm})$ to the source.[22] In our study, TPS is also observed to calculate lower values within the difference of $2.82 \%$ than $\mathrm{MC}$ at $2 \mathrm{~mm}$ distance from the source. This deviation decreases as the distance from the source increases. However, beyond $8 \mathrm{~mm}$ of the source, the TPS calculated values become $0.5 \%$ larger than $\mathrm{MC}$ for all irradiation scenarios. Our results are in good agreement with literature findings. In the present study, TPS calculated doses are lower beyond $3.5 \mathrm{~cm}$ distance from the source. We found larger discrepancies between MC and TPS beyond $10 \mathrm{~cm}$ distance for all the irradiation lengths studied. In the present study, TPS calculates the dose according to the TG-43 report. In this formalism, radial dose function is an important dosimetry parameter that indicates the dose variation by means of photon interactions, such as absorption and scattering in transverse plane. HDR TPSs use the fifth-order polynomial fit for tabulated $g(r)$ data.[5] Chandola et al. calculated the radial dose function of the Nucletron mHDR-v2 Ir-192 source using EGSnrc and Geant 4 codes and measured with the in-air ion chamber.[23] They compared their findings with each other and with the findings by Daskalov.[24] They found good agreement between the MC calculated and measured $g(r)$ values up to $5 \mathrm{~cm}$ distance from the source center. However, the deviation between the obtained $g(r)$ values was observed to slightly increase at further distances from the source. This increased deviation was ascribed to the volume average effect of the ion chamber and various other uncertainties. The deviation between the simulated $g(r)$ values with different 
software packages increases as the distance increases beyond $8 \mathrm{~cm}$ from the source from their tabulated and drawn radial dose function. We also observed the larger discrepancies between the MC and TPS calculated dose rate values on the transverse plane of the irradiation length. The observed largest deviation at $10 \mathrm{~cm}$ away from the source increases as the dwell positions or irradiation length increases. This deviation increase might be attributed to the variation of the radial dose function at large distances from the source.

\section{Conclusion}

Dose rate profiles calculated by Oncentra HDR brachytherapy TPS have been verified with MCNP software for four different irradiation lengths between 2 and $5 \mathrm{~cm}$. There is a good consistency between TPS and MCNP calculated dose rate profiles at distances between $2 \mathrm{~mm}$ and $8 \mathrm{~cm}$ away from the source. The dose difference is obtained within $2 \%$ at above the mentioned distance interval for all the irradiation scenarios. The larger dose difference of approximately $2.8 \%$ was observed at the near source region, and this deviation was found to be independent from the number of dwell positions. The discrepancy between TPS and MC calculated dose rate values increases as the distance from the source increases beyond $5 \mathrm{~cm}$ from the source center, and its amount has been affected by the irradiation length or in another word number of dwell positions. The largest difference of $5.2 \%$ has been found at $10 \mathrm{~cm}$ distance away from the source for $5 \mathrm{~cm}$ irradiation length.

Peer-review: Externally peer-reviewed.

Conflict of Interest: None declared.

Ethics Committee Approval: No ethical approval was required for this study.

Financial Support: None declared.

Authorship contributions: Concept - G.K.; Design H.A.B., A.B., G.K.; Supervision - G.K., A.B.; Materials H.A.B., A.B., G.K.; Data collection \&/or processing - H.A.B., A.B., G.K.; Analysis and/or interpretation - H.A.B., A.B., G.K.; Literature search - H.A.B.; Writing - H.A.B.; Critical review - G.K., A.B.

\section{References}

1. Suntharalingam N, Podgorsak EB, Tölli H. Brachytherapy: Physical and Clinical Aspects. In: Podgorsak EB, Editor. Radiation Oncology Physics: A Handbook for
Teachers and Students. Vienna: International Atomic Energy Agency; 2005. p. 454.

2. Angelopoulos A, Baras P, Sakelliou L, Karaiskos P, Sandilos P. Monte Carlo dosimetry of a new 192Ir high dose rate brachytherapy source. Med Phys 2000;27(11):2521-7.

3. Strohmmaier S, Zwierzchowski G. Comparison of 60Co and 192Ir sources in HDR brachytherapy. J Contemp Brachytherapy 2011;3(4):199-208.

4. Rivard MJ, Venselaar JLM, Beaulieu L. The evolution of brachytherapy treatment planning. Med Phys 2009;36(6):2136-53.

5. Rivard MJ, Coursey BM, DeWerd LA, Hanson WF, Hug MS, Ibbott GS, et al. Update of AAPM Task Group No. 43 Report: A revised AAPM protocol for brachytherapy dose calculations. Med Phys 2004;31(3):633-74.

6. Perez-Calatayud J, Ballester F, Serrano-Andres MA, Puchades V, Lluch JL, Limami Y, et al. Dosimetry characteristics of the Plus and 12i GammaMed PDR 192 Ir sources. Med Phys 2001;28(12):2576-85.

7. Medich DC, Munro JJ 3rd. Monte Carlo characterization of the M-19 high dose rate Iridium-192 brachytherapy source. Med Phys 2007;34(6):1999-2006.

8. Perez-Calatayud J, Cabanero DG, Ballaster FB. Monte Carlo Application in Brachytherapy Dosimetry. In: Lemoigne Y, Caner A, editors. Radiotherapy and Brachytherapy. Netherlands: Springer; 2009. p. 23940.

9. De Boeck L, Belien J, Egyed W. Dose optimization in high-dose-rate brachytherapy: A literature review of quantitative models from 1990 to 2010. Oper Res Health Care 2014;3(2):80-90.

10. Giannouli S, Baltas D, Milickovic N, Lahanas M, Kolotas C, Zamboglou Z, et al. Autoactivation of source dwell positions for HDR brachytherapy treatment planning. Med Phys 2000;27(11):2517-20.

11. Mowlavi AA, Cupardo F, Severgnini M. Monte Carlo and experimental relative dose determination for an Iridium-192 source in water phantom. Iran J Radiat Res 2008;6(1):37-42.

12. Lymperopoulou G, Pantelis E, Papagiannis P, RozakiMavrouli H, Sakelliou L, Baltas D, et al. A Monte Carlo dosimetry study of vaginal 192Ir brachytherapy applications with a shielded cylindrical applicator set. Med Phys 2004;31(11):3080-6.

13. Mosleh Shirazi MA, Faghihi R, Siavashpour Z, Nedaie HA, Mehdizadeh S, Sina S. Independent evaluation of an in-house brachytherapy treatment planning system using simulation, measurement and calculation methods. J Appl Clin Med Phys 2012;13(2):3687.

14. Naseri A, Mesbahi A. Application of Monte Carlo calculations for validation of a treatment planning system in high dose rate brachytherapy. Rep Pract Oncol Radiother 2009;14(6):200-4. 
15. Bahreyni Toossi MT, Abdollahi M, Ghorbani M. A Monte Carlo study on dose distribution validation of GZP6 (60)Co stepping source, Rep Pract Oncol Radiother 2013;18(2):112-6.

16. Hadad K, Zohrevand M, Faghihi R, Sedighi Pashaki A. Accuracy evaluation of OncentraTM TPS in HDR Brachytherapy of Nasopharynx cancer using EGSnrc Monte Carlo code. J Biomed Phys Eng 2015;5(1):25-30.

17. Briesmeister JF. MCNPTM - A general Monte Carlo $\mathrm{N}$-particle transport code, Version 4C. Los Alamos National Laboratory Report. Available at: https:// permalink.lanl.gov/object/tr?what=info:lanl-repo/lareport/LA-13709-M. Accessed February 16, 2019.

18. Ballester F, Puchades V, Lluch JL, Serrano-Andres MA, Limami Y, Perez-Calatayud J, et al. Technical note: Monte-Carlo dosimetry of the HDR $12 \mathrm{i}$ and Plus 192Ir sources. Med Phys 2001;28(12):2586-91.

19. Ballester F, Perez-Calatayud J, Puchades V, Lluch JL, Serrano-Andres MA, Limami Y, et al. Monte Carlo dosimetry of the Buchler high dose rate 192Ir source. Phys Med Biol 2001;46(3):N79-90.

20. Granero D, Vijande J, Ballester F, Rivard MJ. Dosimetry revisited for the HDR 192Ir brachytherapy source model mHDR-v2. Med Phys 2011;38(1):487-94.

21. Almansa Lopez JF, Torres Donaire J, Guerrero Alcalde R. Monte Carlo dosimetry of the most commonly used 192Ir high dose rate brachytherapy sources. Rev Fis Med 2011;12(3):159-68.

22. Wang R, Li XA. Dose characterization in the nearsource region for two high dose rate brachytherapy sources. Med Phys 2002;29(8);1678-86.

23. Chandola RM, Tiwari S, Kowar MK, Choudhary V. Monte Carlo and experimental dosimetric study of the mHDR-v2 brachytherapy source. J Cancer Res Ther 2010;6(4):421-6.

24. Daskalov GM, Löffler E, Williamson JF. Monte Carloaided dosimetry of a new high dose-rate brachytherapy source. Med Phys 1998;25(11):2200-8. 